\title{
EDITORIAL
}

\section{Childhood TB: Situation Analysis and the Potential Solutions}

Tuberculosis (TB) is one of the leading causes of morbidity and mortality of children in tuberculosisendemic areas ${ }^{1}$. Based on vital registration data, World Health Organization (WHO) in 2014, estimated that 1 million children ( $<15$ years) suffer from TB worldwide and 140,000 die each year, representing $10 \%$ and $9 \%$ of global caseload and mortality, respectively ${ }^{2}$. This "merciless disease" exists in the shadow of adult TB and children particularly those under 5 years of age, who came in contact with smear positive adult TB cases and who suffers from malnutrition and HIV infection are the most vulnerable group to acquire tuberculosis ${ }^{3}$. Childhood TB (CTB) remains a neglected aspect of the TB epidemic, despite its contribution to $20 \%$ or more of the TB caseload in many countries with high TB incidence ${ }^{2}$. Among the 4,452,860 new cases reported in 2010 by the 22 highest TB burden countries, only 157,135, i.e. 3.5\% (range, 0.1 to 15.0), were reported as $\mathrm{CTB}^{4}$. However, the best estimates suggest that children (under 15 years of age) account for approximately $11 \%$ of the total TB burden,reflecting that just over 332,000 (7.5\%) of CTB went undiagnosed or unreported in these countries ${ }^{4-5}$.

In light of these facts we can assume that, the actual burden of CTB is likely higher, but is not reflected in data because of challenges in diagnosing the cases. The barriers of diagnosis are related to not obtaining high quality specimens, pauci-bacillary nature of the disease in young children and lack of mycobacterial culture facilities in settings where TB and malnutrition are endemic, even when available, the longtime delay in getting results of culture and sensitivity [6]. Although, the gene X-pert MTB/RIF assay (Cepheid, Sunnyvale, CA, USA) is a new, rapid diagnostic test for the detection of $\mathrm{M}$ tuberculosis than culture it is demonstrating encouraging results in the diagnosis of pulmonary TB especially with greater sensitivity. Still, the sensitivity is less than $70 \%$ compared to culture in children ${ }^{6-8}$.

Reviewing the previous data, we can also postulate that poor ascertainment and reporting of TB casesis another limitation and actually it prevents accurate estimation and true picture of the global burden of $\mathrm{CTB}^{9}$. Many a times, deaths of HIV-infected children with TB is recorded as death due HIV and not as $\mathrm{TB}^{10}$. Similarly, in endemic settings, TB is commonly found in children dying with pneumonia and reported as death from pneumonia not from $\mathrm{TB}^{6,11,12}$.

Despite all these limitations, evidence indicates that global case detection of TB is improving, and it is mainly due to intensified case finding following WHO guideline, notification through a harmonized bridging between government and non-government organizations, scrupulous adherence to national TB guideline, and implementation of DOTs program in many TB-endemic countries ${ }^{2,13,14}$. However, the scenario is not the same in many TB-endemic countries including Bangladesh where under-estimation of childhood TB is still prevailing.

Bangladesh stands 7th amongst the 22 high TB burden countries in the world. As in many high-Tb burden countries, childhood tuberculosis (CTB) is also grossly under-detected in Bangladesh ${ }^{15}$. In 2007, Bangladesh National Tuberculosis Programme (BNTP) reported incidence of childhood TB as 9 per 100000 [16]. In 2011, of the total 155,673 newly reported TB cases, only 4,672 (3\%) cases occurred in childrenunder 15 years ${ }^{17}$. The National incidence of CTB among 0-14 years old children were 9 per 100,000 reported by the NTP in 2007 and 8.6 per 100,000 reported by the Damien Foundation in $2009^{18}$. However, extrapolating data of best estimate (CTB; $11 \%$ of total case load), the estimated incidence and prevalence of CTB is likely to be $25 / 100,000$ and 45 / 100,000 respectively in Bangladesh ${ }^{19}$. A survey conducted by ICDDR,B in 2008-09 in Madhupur, Tangail, showed incidence of CTB as 52 per 100,000 among 0-14 year children which is about 6 times higher than BNTP data $^{20}$. TB prevalence among adults in the same area was estimated at 207/100 000 population, with children representing approximately $20 \%$ of all cases identified. 
Although, this does not represent national incidence of CTB, it definitely indicates a gap between reported and actual disease burden in the communities. Using these numbers, it is estimated that around 21, 000 children with TB remain undetected each year in Bangladesh. The plausible reasons behind this underestimation may be i) poor awareness about childhood TB ii) difficulties in access to TB diagnosis and care iii) clinical similarities with other common childhood diseases iv) Treatment of CTB outside the national TB program v) lack of routine recording and reporting of the cases and most importantly vi) lack of systematic screening for TB among children living in the same households affected by $\mathrm{TB}^{19}$. In addition, recommendations of INH preventive therapy (IPT) for children, under 5 years of age is rarely implemented ${ }^{20}$.

The delay in diagnosis or under-diagnosis of TB among children leave them in jeopardy and often results in serious consequences and fatal outcome ${ }^{21}$. Any child living in a setting where there are people with infectious TB can become ill with TB, even if they are vaccinated. Current TB vaccine protects young children against the most severe forms of $\mathrm{TB}$, such as meningitis and disseminated TB disease, but prevention of transmission from an infectious contact is variable. We do not know the extent to which TB is a cause of childhood deaths because many of them are reported in global statistics as deaths due to HIV, pneumonia, malnutrition or meningitis, but the number is likely to be substantial ${ }^{2}$.

Therefore, to alleviate this situation, strengtheningTB case detection as well as reporting is very important. It is possible through orientation \&training on TBof health workers to understand and improve their clinical skill of TB diagnosis, masspeople awareness through advocacy, counseling and social mobilization, active contact search and mandatory reporting of the cases. In addition, diagnosis and early initiation of treatment of children with TB having associated comorbidities will help to reduce TB related deaths. We need to move beyond the traditional approach of TB care by working synergistically across the entire health system and partnering with communities to reach the goal of zero TB deaths in children.

To achieve that, we need determined leadership, political commitment at all levels, joint efforts by all the stakeholders involved in TB care, relentless and robust research on CTBand of course mobilization of increased resources and this is the demand of time.

[Acknowledgement: Dr. Jobayer Chisti ICDDRB,Dr. Mehdi Parvez SBMC,DrZohoraJameela Khan DMC]

(J Bangladesh Coll Phys Surg 2016; 34: 53-55)

\section{Prof. Md. Abid Hossain Mollah \\ Professor of Pediatrics \\ Dhaka Medical College}

\section{References:}

1. Graham SM, Sismanidis C, Menzies HJ, Marais BJ, Detjen AK, Black RE. Importance of tuberculosis control to address child survival. Lancet. 2014;383:1605-7.

2. World Health Organization. Global tuberculosis report 2015. Geneva, Switzerland: World Health Organization; 2015.

3. World Health Organization. Roadmap for Childhood Tuberculosis”. Geneva: World Health Organization; 2013.

4. Perez-Velez CM, Marais BJ. Tuberculosis in children. N Engl J Med. 2012;367:348-61.

5. Nelson LJ, Schneider E, Wells CD, Moore M. Epidemiology of childhood tuberculosis in the United States, 1993-2001: the need for continued vigilance. Pediatrics. 2004;114:333-41.

6. Chisti MJ, Graham SM, Duke T, Ahmed T, Ashraf H, Faruque AS, et al. A Prospective Study of the Prevalence of Tuberculosis and Bacteraemia in Bangladeshi Children with Severe Malnutrition and Pneumonia Including an Evaluation of Xpert MTB/RIF Assay. PloS one. 2014;9:e93776.

7. Bates M, O’Grady J, Maeurer M, Tembo J, Chilukutu L, Chabala C, et al. Assessment of the Xpert MTB/RIF assay for diagnosis of tuberculosis with gastric lavage aspirates in children in sub-Saharan Africa: a prospective descriptive study. The Lancet infectious diseases. 2013;13:36-42.

8. Rachow A, Clowes P, Saathoff E, Mtafya B, Michael E, Ntinginya EN, et al. Increased and expedited case detection by Xpert MTB/RIF assay in childhood tuberculosis: a prospective cohort study. Clin Infect Dis. 2012;54: 1388-96.

9. Graham SM, Cuevas LE, Jean-Philippe P, Browning R, Casenghi M, Detjen AK, et al. Clinical Case Definitions for Classification of Intrathoracic Tuberculosis in Children: An Update. Clin Infect Dis. 2015;61Suppl 3:S179-87.

10. World Health Organization.Stop TB partnership. Annual meeting of the Childhood TB Subgroup. Kuala Lumpur, Malaysia,: World Health Organization; 2012. 
11. Nantongo JM, Wobudeya E, Mupere E, Joloba M, Ssengooba W, Kisembo HN, et al. High incidence of pulmonary tuberculosis in children admitted with severe pneumonia in Uganda. BMC Pediatr. 2013;13:16.

12. Oliwa JN, Karumbi JM, Marais BJ, Madhi SA, Graham SM. Tuberculosis as a cause or comorbidity of childhood pneumonia in tuberculosis-endemic areas: a systematic review. The Lancet Respiratory medicine. 2015;3: 235-43.

13. Oshi DC, Chukwu JN, Nwafor CC, Meka AO, Madichie NO, Ogbudebe CL, et al. Does intensified case finding increase tuberculosis case notification among children in resourcepoor settings? A report from Nigeria. International journal of mycobacteriology. 2016;5:44-50.

14. Aketi L, Kashongwe Z, Kinsiona C, Fueza SB, Kokolomami J, Bolie G, et al. Childhood Tuberculosis in a Sub-Saharan Tertiary Facility: Epidemiology and Factors Associated with Treatment Outcome. PloS one. 2016;11:e0153914.

15. Rashid MM. Tuberculosis and Malaria Control Programme in Bangladesh.: World Bank Group. Public Private Dialogue. 8th PPD Workshop. 2015.

16. National Tuberculosis Control Programme. Tuberculosis control Increasing child TB detection in Bangladesh.
Annual report 2008. Dhaka, Bangladesh: . Directorate General of Health Services. 2008.

17. National Guideline for the Management of Tuberculosis in Children. Second ed2016.

18. National Guideline for the Management of Tuberculosis in Children. First ed2012.

19. Bangladesh National Tuberculosis programe. Childhood Tuberculosis: A training manual for medical doctors. 2013.

20. Ahmed T, Rahman AS, Islam R. Results from field: novel diagnostics in children 41st World Conference on Lung Health of the International Union Against Tuberculosis and Lung Disease (The Union), 11- 15 November 2010. Berlin, Germany: Int J Tuberc Lung Dis; 2010. p. S34-S5.).

21. Yan I, Korenromp E, Bendavid E. Mortality changes after grants from the Global Fund to Fight AIDS, tuberculosis and malaria: an econometric analysis from 1995 to 2010. BMC public health. 2015;15:977.

22. Chisti MJ, Ahmed T, Pietroni MA, Faruque AS, Ashraf H, Bardhan PK, et al. Pulmonary tuberculosis in severelymalnourished or HIV-infected children with pneumonia: a review. Journal of health, population, and nutrition. 2013;31:308-13. 